\title{
An analysis of partial efficiencies of energy utilisation of different macronutrients by barramundi (Lates calcarifer) shows that starch restricts protein utilisation in carnivorous fish
}

\author{
Brett D. Glencross ${ }^{1 * \dagger}$ David Blyth $^{2}$, Nicholas Bourne ${ }^{1}$, Susan Cheers ${ }^{1}$, Simon Irvin $^{2}$ and Nicholas M. Wade ${ }^{1}$ \\ ${ }^{1}$ Commonwealth Scientific and Industrial Research Organisation (CSIRO) Agriculture, Queensland Biosciences Precinct, St \\ Lucia, Qld 4067, Australia \\ ${ }^{2}$ CSIRO Agriculture, Bribie Island Research Centre, Woorim, Qld 4507, Australia
}

(Submitted 16 February 2016 - Final revision received 1 December 2016 - Accepted 25 January 2017 - First published online 14 March 2017)

\section{Abstract}

This study examined the effect of including different dietary proportions of starch, protein and lipid, in diets balanced for digestible energy, on the utilisation efficiencies of dietary energy by barramundi (Lates calcarifer). Each diet was fed at one of three ration levels (satiety, $80 \%$ of initial satiety and $60 \%$ of initial satiety) for a 42 -d period. Fish performance measures (weight gain, feed intake and feed conversion ratio) were all affected by dietary energy source. The efficiency of energy utilisation was significantly reduced in fish fed the starch diet relative to the other diets, but there were no significant effects between the other macronutrients. This reduction in efficiency of utilisation was derived from a multifactorial change in both protein and lipid utilisation. The rate of protein utilisation deteriorated as the amount of starch included in the diet increased. Lipid utilisation was most dramatically affected by inclusion levels of lipid in the diet, with diets low in lipid producing component lipid utilisation rates well above $1 \cdot 3$, which indicates substantial lipid synthesis from other energy sources. However, the energetic cost of lipid gain was as low as $0.65 \mathrm{~kJ}$ per $\mathrm{kJ}$ of lipid deposited, indicating that barramundi very efficiently store energy in the form of lipid, particularly from dietary starch energy. This study defines how the utilisation efficiency of dietary digestible energy by barramundi is influenced by the macronutrient source providing that energy, and that the inclusion of starch causes problems with protein utilisation in this species.

Key words: Energetics: Proteins: Starch: Lipids: Asian seabass

Barramundi is an obligate carnivorous fish species that forms the basis of a significant aquaculture industry in south-east Asia and Australia $^{(1)}$. The development of high-nutrient density, formulated extruded feeds has been underpinned by the development of both a series of factorial bioenergetic nutritional models and foundation empirical studies ${ }^{(1-5)}$. These nutritional models have so far relied on the assumption that the dietary digestible energy (DE) source is irrelevant - that is, the dietary DE derived from protein, lipid and starch is utilised with equal efficiency, subject to key nutrients (e.g. protein) being provided at/or above minimum critical ratios to energy supply ${ }^{(4-10)}$.

Each of the different macronutrients (starch, protein and lipid) supplies energy by distinct metabolic pathways. In aquatic animals, it is recognised that there are different levels of efficiency in the utilisation of each of these macronutrients for energy $^{(11,12)}$. It is now recognised that this difference requires an amendment of the digestible nutritional values of each macronutrient to those of metabolisable nutritional values and/or net energy (NE) nutritional values ${ }^{(9,12-14)}$. A recent study by Schrama et $a l .{ }^{(14)}$ examined the utilisation of both starch and lipid for growth by the omnivorous fish Nile tilapia (Oreochromis niloticus). These authors observed that each macronutrient had a different effect on the partial efficiencies of utilisation of $\mathrm{DE}\left(k_{D E}\right)$ by the fish, with dietary utilisation coefficients of 0.561 and 0.663 being observed for starch- and lipid-based diets, respectively. These observations clearly indicated that this fish species used lipid as an energy source for growth more efficiently. However, the third key macronutrient, protein, was not considered in this study. In the same study, Schrama et $a l .{ }^{(14)}$ by reviewing the literature identified that there was a wide variability $(0 \cdot 31-0 \cdot 82)$ in the $k_{D E}$ of different studies. It was suggested that the three primary reasons for this variability were as follows: different dietary macronutrient compositions, trophic level of the fish species and the composition of growth. In addition, there is increasing evidence that the roles of gluconeogenesis, glycolysis and $\beta$-oxidation play substantially different relative roles in energy provision in fish compared with other vertebrates ${ }^{(11,14-17)}$.

Abbreviations: DE, digestible energy; DEI, digestible energy intake; HEm, maintenance energy demands; $\boldsymbol{k}_{E}$, coefficient of utilisation; MEI, metabolisable energy intake; RE, retained energy.

* Corresponding author: B. D. Glencross, email B.D.Glencross@stir.ac.uk

$\dagger$ Present address: Institute of Aquaculture, Stirling University, Stirling, UK. 
The objective of this study was to determine the partial efficiencies of utilisation of each of the different diets based on equivalent DE densities, but differing in ratio of each of the macronutrient energy substrates. By using a diet-by-ration factorial study, it was proposed that it would be possible to not only derive the partial efficiencies for each diet, but also by overlaying a multiple regression analysis of the responses to derive the discrete partial energetic efficiencies for each of the macronutrients. By determining these responses, it will help provide evidence for the true energetic role that each of the three macronutrients (protein, lipid and starch) plays as an energy source in diets when fed to barramundi.

\section{Methods \\ Diet preparation}

The diets used in this study were based on equivalent DE densities, but differed in the ratio of each of the macronutrient energy substrates. From this design, it will be possible to not only derive the partial efficiencies for each diet, but also by overlaying a multiple regression analysis of the responses to derive the discrete partial energetic efficiencies for each of the macronutrients used within each diet. The diets used in this study are based on diets used in the earlier study by Glencross et $a l^{(12)}$. In this experiment, each of the diets was formulated to be isoenergetic $(15.3 \mathrm{MJ} \mathrm{DE} / \mathrm{kg})$ on a digestible nutrient basis based on the ingredient digestibility values determined by Glencross et $a l .{ }^{(12)}$. Most diets were also isoproteic $(475 \mathrm{~g} / \mathrm{kg}$ ) on a digestible basis, with the exception of the 'P' diet, in which the digestible protein was $562 \mathrm{~g} / \mathrm{kg}$. An additional diet (C) was used as reference for diet specifications typically used in commercial diets.

Diets were prepared by mixing all the dry ingredients, and then processed by addition of oil and water (about $30 \%$ of mash dry weight) while mixing to form a dough. The dough was then screw-pressed through a 4-mm diameter die using a pasta maker (Dolly; La Monferrina). The resultant moist pellets were oven-dried at $65^{\circ} \mathrm{C}$ for $12 \mathrm{~h}$ before being air-cooled, bagged and stored at $-20^{\circ} \mathrm{C}$. Formulations and composition of the diets are presented in Table 1.

\section{Fish handling}

All animal procedures were approved by the CSIRO Animal Ethics Committee (approval no. A9/2011). Juvenile barramundi (Lates calcarifer) were obtained from a commercial hatchery (Betta Barra), and were on-grown to 69.6 (sD 0.75) g ( $n$ 480) in preparation for the experiment. During the on-growing period, all fish were fed the same diet (Marine Float; Ridley Aquafeeds) and maintained in $2 \times 5000$-litre seawater tanks. At trial initiation, forty fish were weighed on an electronic top-loading balance to $0 \cdot 1 \mathrm{~g}$ accuracy to determine the mean and standard deviation of the population. Following this, twenty fish were allocated to $24 \times 300$-litre tanks on the basis of having to be within the mean (SD 1). The experiment was conducted at the Bribie Island Research Centre at Woorim, in a flow-through (3litre/min), aerated, heated seawater tank array. Water temperature was maintained at mean $29.9(\mathrm{SD} 0 \cdot 12)^{\circ} \mathrm{C}$, and the
Table 1. Formulation, composition and relative digestible contributions of the energy of each macronutrient in each of the experimental diets

\begin{tabular}{|c|c|c|c|c|}
\hline & C & $\mathrm{P}$ & $\mathrm{L}$ & $\mathrm{S}$ \\
\hline \multicolumn{5}{|l|}{ Diet formulations } \\
\hline Fishmeal (Anchovetta) & 560 & 640 & 560 & 560 \\
\hline Wheat gluten & 100 & 100 & 100 & 100 \\
\hline Casein & 50 & 100 & 50 & 50 \\
\hline Fish oil (Anchovetta) & 50 & 40 & 100 & 0 \\
\hline Pregelatinised wheat starch & 120 & 0 & 0 & 240 \\
\hline Yttrium oxide & 2 & 2 & 2 & 2 \\
\hline Vitamin-mineral premix & 5 & 5 & 5 & 5 \\
\hline Cellulose & 113 & 113 & 183 & 43 \\
\hline \multicolumn{5}{|l|}{ Diet composition } \\
\hline DM & 974 & 975 & 945 & 909 \\
\hline Crude protein & 505 & 603 & 483 & 493 \\
\hline Digestible protein & 448 & 545 & 455 & 441 \\
\hline Total lipid & 107 & 107 & 148 & 68 \\
\hline Digestible lipid & 107 & 94 & 148 & 67 \\
\hline Ash & 108 & 122 & 104 & 104 \\
\hline Total carbohydrates & 280 & 169 & 264 & 336 \\
\hline Total starch & 135 & 17 & 29 & 225 \\
\hline Digestible starch & 111 & 13 & 29 & 214 \\
\hline Gross energy (kJ/g DM) & $21 \cdot 39$ & $20 \cdot 24$ & 20.69 & $20 \cdot 71$ \\
\hline Digestible energy (kJ/g DM) & $16 \cdot 61$ & $16 \cdot 70$ & $16 \cdot 91$ & $16 \cdot 69$ \\
\hline Digestible energy as protein (\%) & $63 \cdot 6$ & $76 \cdot 5$ & 63.4 & $62 \cdot 4$ \\
\hline Digestible energy as lipid (\%) & $24 \cdot 8$ & $21 \cdot 5$ & $33 \cdot 6$ & $15 \cdot 5$ \\
\hline Digestible energy as starch (\%) & $11 \cdot 6$ & $2 \cdot 0$ & $3 \cdot 0$ & $22 \cdot 2$ \\
\hline
\end{tabular}

C, control; P, protein; L, lipid; S, starch.

dissolved oxygen level was 5.5 (SD 0.56 ) $\mathrm{mg} / \mathrm{l}$ for the $42-\mathrm{d}$ experiment

Diets were manually supplied to the tanks. Three ration levels were used - a satiety level, $80 \%$ of the initial satiety level and $60 \%$ of the initial satiety level. The satiety rations were fed twice daily at $09.00-09.30$ hours and $16.30-17.00$ hours. Satiety levels were determined by feeding to slight excess all diets, and uneaten feed was accounted for. Correction factors were applied to determine solubilisation losses and pellet DM. The actual feed consumption within each tank was thus calculated on the basis of the methods reported by Helland et al. ${ }^{(18)}$. The two restricted rations used in this study were based on 80 and $60 \%$ of the measured initial demand, which was also consistent with the model of Glencross ${ }^{(4)}$. These rations were not adjusted over time. Each treatment was duplicated within the 24-tank array. On the basis of the plan for using regression analysis in this experiment, it was proposed that a 3 rations $\times 2$ replicates design is stronger than a 2 rations $\times 3$ replicates approach.

\section{Sample preparation and chemical analysis}

Five fish were euthanised from the population at the beginning of the experiment as a representative initial sample. At the end of experiment, five fish from each tank were euthanised by immersion in an overdose of AQUI-S ${ }^{\mathrm{TM}}$ (AQUI-S) before being placed in an iced-seawater slurry. Following sample collection, each whole-fish sample was frozen before being minced using an industrial food processor to ensure sample homogeneity. Samples were then collected, and their moisture content was determined by oven drying at $105^{\circ} \mathrm{C}$ for $24 \mathrm{~h}$, and a second sample was freeze-dried for chemical analysis. Freeze-dried fish samples were milled before analysis for DM, ash, fat, $\mathrm{N}$ and 
gross energy contents. Diet and faecal samples were analysed for DM, yttrium, N, lipid, starch and gross energy contents.

DM was calculated by gravimetric analysis following oven drying at $105^{\circ} \mathrm{C}$ for $24 \mathrm{~h}$. Total yttrium concentrations were determined after mixed acid digestion using inductively coupled plasma-MS. Protein levels were calculated by determining total $\mathrm{N}$ using a CHNOS auto-analyser (Thermo Fisher Scientific), based on $\mathrm{N} \times 6 \cdot 25$. Total starch content of the diets was measured using an enzymatic method with the Total Starch Kit K-TSTA (Megazyme), following a modified Association of Official Analytical Chemists Method 996.11. Total lipid content of the diets was determined gravimetrically following extraction of the lipids using chloroform-methanol (2:1). Gross ash content was determined gravimetrically following loss of mass by combustion of a sample in a muffle furnace at $550^{\circ} \mathrm{C}$ for $12 \mathrm{~h}$. Gross energy was determined by ballistic bomb calorimetry. All analyses were performed in accordance with the specifications of Association of Official Analytical Chemists ${ }^{(19)}$.

\section{Diet digestibility analysis}

At the end of the growth experiment and following sample collection, the remaining fish in the eight tanks fed to satiety were used for faeces collection. Fish were subjected to faecal stripping once daily about $6 \mathrm{~h}$ after feeding. Faecal stripping was carried out on the basis of the methods reported by Blyth et al. ${ }^{(20)}$. This involved netting of fish into a separate tank and rapid sedation to induce muscle relaxation. Once muscle relaxation occurred, fish were removed from the anaesthetic-containing water, stripped with gentle, manual abdominal pressure and faecal matter was expelled into a collection jar. Fish were then returned to their original tanks for recovery. Faeces samples were collected over a minimum of three stripping events, pooled within each tank and kept frozen for future analysis.

Differences in the ratios of DM, protein, lipid (insufficient faecal sample was available for starch analysis) or gross energy: yttrium in the feed and faeces for each treatment were calculated to determine the apparent digestibility $\left(A D_{\text {diet }}\right)$ for each of the nutritional parameters examined in each diet based on the following formula:

$$
A D_{\text {diet }}=\left(1-\left(\frac{Y_{\text {diet }} \times \text { Parameter }_{\text {faeces }}}{Y_{\text {faeces }} \times \text { Parameter }_{\text {diet }}}\right)\right) \times 100,
$$

where $Y_{\text {diet }}$ and $Y_{\text {faeces }}$ represent the yttrium content of the diet and faeces, respectively, and Parameter Piet $_{\text {and }}$ arameter faeces represent the nutritional parameters of concern (DM, protein or energy) in diet and faeces, respectively.

\section{Protein and energy utilisation analysis}

Protein $(\mathrm{N} \times 6.25)$ and energy (E) utilisation were determined on the basis of gain in both $\mathrm{N}$ and $\mathrm{E}$ over the experimental period, against the respective consumption of digestible $\mathrm{N}$ and $\mathrm{E}$ over the experimental period. Both gain and intake values were calculated on the basis of daily gain amount per unit body weight. To provide independence of size effects, modelling of protein, lipid and energy utilisation data was carried out with respect to known protein, lipid and energy body weight exponents for barramundi of $x^{0.7}, x^{0.9}$ and $x^{0.8}$, respectively ${ }^{(21,22)}$. Both protein energy and lipid energy utilisation were transformed to the energy body weight exponent value of $x^{0 \cdot 8}$.

\section{Nutrient and energy balance and deposition assessment}

The net balances for protein (P), lipid (L) and energy (E) were calculated on the basis of the data derived from this study. The methods used for these calculations were based on those reported by Saravanan et al. ${ }^{(11)}$. Gross intake levels of each nutrient were determined on the basis of total feed intake for each tank multiplied by the percent composition of the feed being fed. Digestible intake levels were measured similarly on the basis of the digestibility of P, L and E from each diet. Faecal losses were determined as the reciprocal of the digestible levels. Retained nutrients and energy were determined on the basis of the net gain in nutrients and energy between fish at the end of the trial and those from the initial sample. Branchial and urinary $\mathrm{N}$ (BUN) were determined on the basis of the difference between digestible $\mathrm{N}$ intake and retained $\mathrm{N}$ with energy values based on $24.85 \mathrm{~kJ} \times$ BUN using values reported by Saravanan et $a l^{(11)}$. The metabolisable energy intake (MEI) was determined on the basis of the digestible energy intake (DEI) minus the branchial and urinary energy losses. Heat production (HP) was determined on the basis of the difference between metabolisable energy and retained energy (RE). Basal metabolism (HeE) was calculated on the basis of the reported fasting energy losses of $34.4 \mathrm{~kJ} / \mathrm{kg}^{0 \cdot 8}$ per $\mathrm{d}^{(4)}$. The heat increment energy (HiE) was determined as follows - MEI minus RE and HeE. NE was determined as MEI minus $\mathrm{HiE}^{(23)}$.

\section{Statistical analysis}

All values are means with their standard errors unless otherwise specified. Effects of diet treatment and ration levels were examined by multivariate ANOVA using the software package Statistica $\left(\right.$ StatSoft $\left.{ }^{\circledR}\right)$. Levels of significance were determined using Fisher's least significant difference test for planned comparisons, with critical limits being set at $P<0.05$. Regression figures presented were constructed using Microsoft Excel. Error terms for linear functions were determined using the regression feature of the Data Analysis package within Microsoft Excel. Multiple regression analysis was used to determine the component energy utilisation parameters based on having definitive assessments of protein energy utilisation efficiencies for each diet, which then enabled derivation, by multiple regression, of the contribution of both lipid energy and starch energy to the partial efficiency of energy utilisation in each diet ${ }^{(24)}$.

\section{Results}

Effect of macronutrient energy bias on growth and body composition

There were significant differences between the effect of diets and feed ration levels on final weight, weight gain, feed intake and feed conversion ratio (FCR) (Table 2). Significant interaction effects on feed intake were observed between diet and 


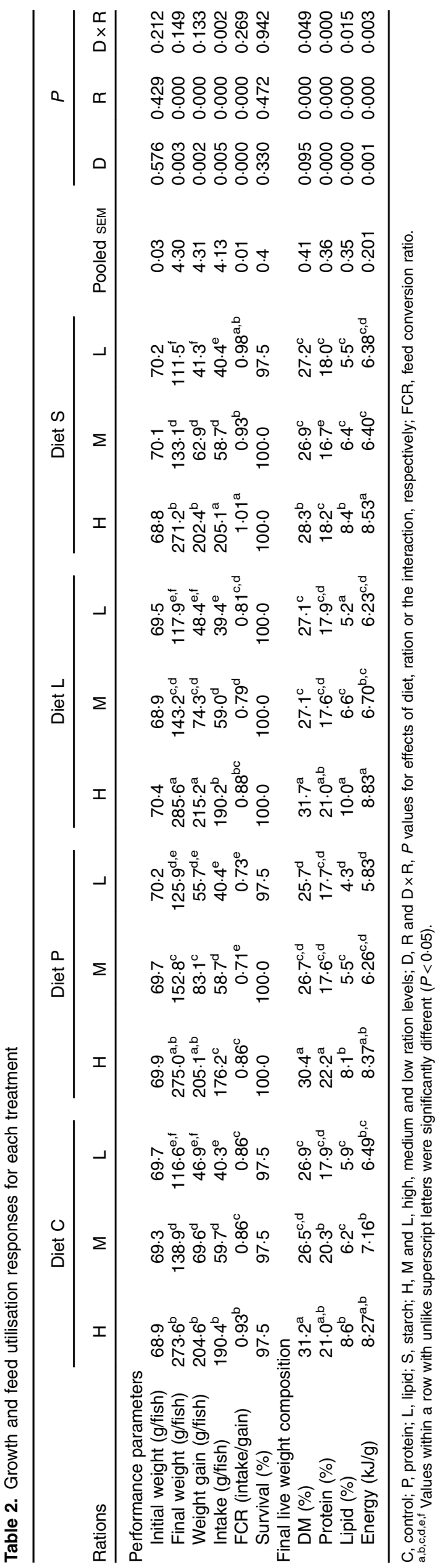

ration levels, but none of the other performance parameters. There were no significant effects on survival attributable to diet, ration or the interaction term. Among fish fed to satiety, weight gain was the highest in fish fed Diet $\mathrm{L}$ and the lowest in fish fed Diet S. However, among fish fed to satiety, feed conversion was the highest in fish fed Diet P and the lowest in fish fed Diet S. Among fish fed to satiety, there were some significant differences in feed intake, with the highest intake in fish fed Diet $S$ and the lowest in fish fed Diet P (Table 2).

There was a significant effect of both feed ration level and diet on final live weight protein concentration, lipid concentration and energy content (Table 2). No significant differences were observed of diet on final live weight DM composition (Table 2). There were also significant interaction terms between diet and ration level on each of the parameters of final live weight DM, protein, lipid and energy concentrations. Key compositional differences were noted in fish fed Diet P, which had less lipid concentrations compared with fish fed Diet L. This effect was most notable at lower fixed ration levels (Table 2).

\section{Effect of macronutrient energy bias on energy utilisation}

The pairwise comparison within feed ration levels between each dietary treatment showed significantly different levels of energy retention between the starch diet and every other treatment (Table 3$)$. The energy utilisation efficiencies $\left(\mathrm{kJ} / \mathrm{kg}^{0 \cdot 8}\right.$ per d) for each diet were described by the following linear equations (Fig. 1):

$$
\begin{gathered}
y_{S}=0.508( \pm 0.010) x-8.859( \pm 2.496), R^{2}=0.998, \\
y_{L}=0.730( \pm 0.023) x-29.821( \pm 5.461), R^{2}=0.996 \\
y_{P}=0.715( \pm 0.012) x-26.324( \pm 2.774), R^{2}=0.999, \\
y_{C}=0.607( \pm 0.015) x-8.686( \pm 3.717), R^{2}=0.997 .
\end{gathered}
$$

The coefficient of utilisation $\left(k_{E}\right)$ was significantly lower for Diet $S$ relative to each of the other diets. Similarly, the utilisation coefficient for Diet C was also significantly lower than that of Diets $\mathrm{P}$ and L. There was no difference in energy utilisation coefficients between Diets $P$ and L. Maintenance energy demands (HEm) were calculated by extrapolating the linear regression to the intercept of the $x$-axis. From this, the following HEm values were derived - Diet S: $17 \cdot 4$ (SEM 0.81) $\mathrm{kJ} / \mathrm{kg}^{0 \cdot 8}$ per d, Diet L: 40.8 (sem 0.98) kJ/kg ${ }^{0 \cdot 8}$ per d, Diet P: $36 \cdot 8$ (sem 0.59) kJ/ $\mathrm{kg}^{0 \cdot 8}$ per d and Diet C: 14.3 (SEM $1 \cdot 14$ ) $\mathrm{kJ} / \mathrm{kg}^{0.8}$ per d. There were significant differences in the HEm values between Diets L and P relative to Diets $\mathrm{S}$ and $\mathrm{C}$, but not within those pairings.

\section{Effect of macronutrient energy bias on protein and lipid energy utilisation}

The pairwise comparison within feed ration levels between each dietary treatment also showed significantly different levels of protein energy retention between starch diet and every other treatment (Table 3). The protein energy utilisation efficiencies 
Table 3. Protein (g/fish), lipid (g/fish) and energy (kJ/fish) balance analysis

\begin{tabular}{|c|c|c|c|c|c|c|c|c|c|c|c|c|c|c|c|c|}
\hline \multirow[b]{2}{*}{ Rations } & \multicolumn{3}{|c|}{ Diet C } & \multicolumn{3}{|c|}{ Diet $P$} & \multicolumn{3}{|c|}{ Diet L } & \multicolumn{3}{|c|}{ Diet S } & \multirow[b]{2}{*}{ Pooled SEM } & \multicolumn{3}{|c|}{$P$} \\
\hline & $\mathrm{H}$ & M & L & $\mathrm{H}$ & $M$ & L & $\mathrm{H}$ & M & L & $\mathrm{H}$ & $\mathrm{M}$ & L & & D & $\mathrm{R}$ & $\mathrm{D} \times \mathrm{R}$ \\
\hline GPI & $96 \cdot 2^{\mathrm{a}}$ & $30 \cdot 2^{\mathrm{b}}$ & $20 \cdot 3^{c}$ & $106 \cdot 3^{a}$ & $35 \cdot 4^{b}$ & $24 \cdot 4^{\mathrm{C}}$ & $91 \cdot 9^{\mathrm{a}}$ & $28 \cdot 5^{\mathrm{b}}$ & $19 \cdot 0^{c}$ & $101 \cdot 1^{\mathrm{a}}$ & $28.9^{\mathrm{b}}$ & $19 \cdot 9^{c}$ & $7 \cdot 27$ & 0.000 & 0.000 & 0.032 \\
\hline FP & $10 \cdot 8^{\mathrm{b}}$ & $3 \cdot 4^{\mathrm{d}}$ & $2 \cdot 3^{\mathrm{e}}$ & $14.2^{\mathrm{a}}$ & $4.7^{c}$ & $3.3^{\mathrm{d}}$ & $5 \cdot 4^{\mathrm{C}}$ & $1.7^{\mathrm{e}}$ & $1.1^{\mathrm{e}}$ & $10 \cdot 6^{\mathrm{b}}$ & $3.0^{\mathrm{d}}$ & $2 \cdot 1^{e}$ & 0.86 & 0.000 & 0.000 & 0.000 \\
\hline DPI & $85 \cdot 4^{\mathrm{a}}$ & $26 \cdot 8^{\mathrm{b}}$ & $18 \cdot 1^{b, c}$ & $92 \cdot 0^{\mathrm{a}}$ & $30.7^{b}$ & $21 \cdot 1^{\mathrm{b}, \mathrm{c}}$ & $86 \cdot 5^{\mathrm{a}}$ & $26 \cdot 8^{\mathrm{b}}$ & $17 \cdot 9^{c}$ & $90 \cdot 5^{\mathrm{a}}$ & $25 \cdot 9^{b}$ & $17 \cdot 8^{\mathrm{c}}$ & $6 \cdot 50$ & 0.002 & 0.000 & 0.246 \\
\hline $\mathrm{BUN}(\mathrm{Peq})$ & $40 \cdot 2^{\mathrm{b}}$ & $11.0^{\mathrm{d}}$ & $9 \cdot 6^{\mathrm{e}}$ & $43 \cdot 5^{\mathrm{a}, \mathrm{b}}$ & $16 \cdot 3^{\mathrm{c}}$ & $11.0^{\mathrm{d}}$ & $39.1^{\mathrm{b}}$ & $13 \cdot 9^{\mathrm{c}, \mathrm{d}}$ & $9.3^{\mathrm{e}}$ & $53 \cdot 5^{\mathrm{a}}$ & $16 \cdot 3^{\mathrm{c}}$ & $10 \cdot 3^{\mathrm{d}, \mathrm{e}}$ & 3.25 & 0.000 & 0.000 & 0.001 \\
\hline $\mathrm{RP}$ & $45 \cdot 2^{a, b}$ & $15 \cdot 8^{\mathrm{c}}$ & $8 \cdot 4^{\mathrm{d}}$ & $48 \cdot 5^{\mathrm{a}, \mathrm{b}}$ & $14 \cdot 3^{\mathrm{c}}$ & $10 \cdot 1^{\mathrm{c}, \mathrm{d}}$ & $47 \cdot 4^{\mathrm{a}}$ & $12 \cdot 9^{\mathrm{a}}$ & $8 \cdot 6^{\mathrm{c}, \mathrm{d}}$ & $37.0^{\mathrm{b}}$ & $9 \cdot 6^{\mathrm{c}, \mathrm{d}}$ & $7 \cdot 5^{\mathrm{d}}$ & 3.38 & 0.000 & 0.000 & 0.000 \\
\hline RP:DPI & $53 \%^{b}$ & $59 \%^{\mathrm{a}}$ & $47 \%^{\mathrm{c}}$ & $53 \%^{b}$ & $47 \%^{\mathrm{c}}$ & $48 \% \%^{b, c}$ & $55 \%{ }^{a, b}$ & $48 \%$ b,c & $48 \%$ b,c & $41 \%^{d}$ & $37 \%^{d}$ & $42 \%^{d}$ & $1.3 \%$ & 0.000 & 0.016 & 0.005 \\
\hline GLI & $20 \cdot 4^{\mathrm{b}}$ & $6 \cdot 4^{\mathrm{d}, \mathrm{e}}$ & $4 \cdot 3^{e, f}$ & $18 \cdot 7^{\mathrm{b}}$ & $6 \cdot 2^{\mathrm{e}}$ & $4 \cdot 3^{\mathrm{e}, \mathrm{f}}$ & $28 \cdot 1^{\mathrm{a}}$ & $8 \cdot 7^{d}$ & $5 \cdot 8^{\mathrm{e}}$ & $13 \cdot 7^{c}$ & $3.9^{f}$ & $2 \cdot 7^{f}$ & 1.62 & 0.000 & 0.000 & 0.000 \\
\hline $\mathrm{FL}$ & $0.1^{\mathrm{c}}$ & $0.0^{\mathrm{c}}$ & $0.0^{\mathrm{c}}$ & $2 \cdot 1^{\mathrm{a}}$ & $0.7^{b}$ & $0.5^{\mathrm{b}}$ & $0.3^{\mathrm{b}, \mathrm{c}}$ & $0.1^{\mathrm{c}}$ & $0.1^{\mathrm{c}}$ & $0.1^{\mathrm{c}}$ & $0.0^{\mathrm{c}}$ & $0.0^{\mathrm{c}}$ & 0.12 & 0.000 & 0.000 & 0.000 \\
\hline DLI & $20 \cdot 3^{b}$ & $6 \cdot 4^{d}$ & $4 \cdot 3^{d}$ & $16 \cdot 6^{b, c}$ & $5 \cdot 5^{\mathrm{d}}$ & $3 \cdot 8^{\mathrm{d}}$ & $27 \cdot 9^{\mathrm{a}}$ & $8 \cdot 6^{\mathrm{c}, \mathrm{d}}$ & $5 \cdot 8^{d}$ & $13 \cdot 7^{\mathrm{c}}$ & $3 \cdot 9^{\mathrm{d}}$ & $2 \cdot 7^{d}$ & 1.59 & 0.000 & 0.000 & 0.000 \\
\hline $\mathrm{RL}$ & $20 \cdot 2^{a, b}$ & $5 \cdot 2^{\mathrm{c}}$ & $3 \cdot 5^{\mathrm{c}}$ & $19 \cdot 0^{\mathrm{b}}$ & $5 \cdot 0^{c}$ & $2 \cdot 0^{c}$ & $25 \cdot 3^{\mathrm{a}}$ & $6 \cdot 1^{\mathrm{c}}$ & $2 \cdot 8^{\mathrm{c}}$ & $19 \cdot 5^{\mathrm{b}}$ & $5 \cdot 2^{\mathrm{c}}$ & $2 \cdot 8^{\mathrm{c}}$ & 1.71 & 0.000 & 0.000 & 0.000 \\
\hline RL:DLI & $99 \%^{\mathrm{b}}$ & $82 \%^{c}$ & $81 \%^{c}$ & $114 \%^{\mathrm{b}}$ & $91 \%$ b,c & $53 \%{ }^{e}$ & $91 \%$ b,c & $71 \%{ }^{\mathrm{c}, \mathrm{d}}$ & $49 \%{ }^{\mathrm{e}}$ & $142 \%^{a}$ & $132 \%^{a}$ & $103 \%^{b}$ & $5.7 \%$ & 0.000 & 0.000 & 0.018 \\
\hline $\mathrm{RL}: \mathrm{RP}$ & $45 \%^{\mathrm{b}}$ & $33 \%^{c}$ & $42 \%^{b}$ & $39 \%$ & $35 \%^{\mathrm{c}}$ & $20 \%{ }^{d}$ & $53 \%^{a}$ & $47 \%^{a, b}$ & $33 \%{ }^{c}$ & $53 \%^{a}$ & $53 \%^{a}$ & $37 \%^{\mathrm{c}}$ & $2.1 \%$ & 0.000 & 0.000 & 0.001 \\
\hline GEI & $4074^{\mathrm{a}}$ & $1278^{b}$ & $862^{b}$ & $3566^{a}$ & $1188^{\mathrm{b}}$ & $818^{b}$ & $3935^{a}$ & $1221^{b}$ & $814^{\mathrm{b}}$ & $4249^{a}$ & $1216^{b}$ & $837^{\mathrm{b}}$ & 291.4 & 0.000 & 0.000 & 0.001 \\
\hline $\mathrm{FE}$ & $910^{\mathrm{a}}$ & $285^{c}$ & $193^{c, d}$ & $624^{\mathrm{b}}$ & $208^{c}$ & $143^{d}$ & $718^{\mathrm{b}}$ & $193^{\mathrm{cd}}$ & $149^{d}$ & $908^{\mathrm{a}}$ & $260^{c}$ & $179^{\mathrm{c}, \mathrm{d}}$ & 60.4 & 0.000 & 0.000 & 0.000 \\
\hline DEI & $3164^{a}$ & $992^{b}$ & $669^{c}$ & $2942^{\mathrm{a}}$ & $980^{\mathrm{b}}$ & $674^{\mathrm{C}}$ & $3217^{a}$ & $1027^{b}$ & $666^{c}$ & $3341^{a}$ & $956^{\mathrm{b}}$ & $658^{\mathrm{c}}$ & $232 \cdot 3$ & 0.023 & 0.000 & 0.006 \\
\hline BUE & $160^{\mathrm{b}}$ & $44^{\mathrm{c}, \mathrm{d}}$ & $38^{\mathrm{d}}$ & $173^{a, b}$ & $65^{\mathrm{c}}$ & $44^{\mathrm{a}}$ & $156^{\mathrm{b}}$ & $55^{\mathrm{c}, \mathrm{d}}$ & $37^{\mathrm{d}}$ & $213^{\mathrm{a}}$ & $65^{\mathrm{c}}$ & $41^{\mathrm{d}}$ & 12.9 & 0.000 & 0.000 & 0.001 \\
\hline MEI & $3004^{\mathrm{a}}$ & $948^{b}$ & $631^{b}$ & $2769^{a}$ & $915^{\mathrm{b}}$ & $631^{\mathrm{b}}$ & $3061^{a}$ & $972^{\mathrm{a}}$ & $629^{b}$ & $3128^{a}$ & $891^{b}$ & $617^{b}$ & 219.7 & 0.011 & 0.000 & 0.005 \\
\hline RE & $1841^{a, b}$ & $572^{\mathrm{c}}$ & $333^{\mathrm{c}, \mathrm{d}}$ & $1875^{a, b}$ & $531^{c}$ & $306^{d}$ & $2090^{a}$ & $540^{c}$ & $311^{d}$ & $1621^{b}$ & $424^{\mathrm{c}, \mathrm{d}}$ & $284^{d}$ & 144.7 & 0.000 & 0.000 & 0.000 \\
\hline $\mathrm{HP}$ & $1163^{b}$ & $377^{\mathrm{d}, \mathrm{e}}$ & $298^{e}$ & $895^{\mathrm{c}}$ & $384^{\mathrm{d}, \mathrm{e}}$ & $324^{\mathrm{d}, \mathrm{e}}$ & $971^{\mathrm{b}, \mathrm{c}}$ & $432^{d}$ & $318^{\mathrm{d}, \mathrm{e}}$ & $1507^{a}$ & $467^{d}$ & $333^{\mathrm{d}, \mathrm{e}}$ & 81.4 & 0.000 & 0.000 & 0.000 \\
\hline $\mathrm{HeE}$ & $295^{a}$ & $226^{\mathrm{b}, \mathrm{c}}$ & $211^{\mathrm{c}}$ & $297^{a}$ & $235^{\mathrm{b}}$ & $218^{b, c}$ & $303^{\mathrm{a}}$ & $228^{\mathrm{b}, \mathrm{c}}$ & $211^{\mathrm{c}}$ & $294^{a}$ & $223^{b, c}$ & $208^{\mathrm{c}}$ & 7.8 & 0.001 & 0.000 & 0.117 \\
\hline $\mathrm{HiE}$ & $868^{\mathrm{b}}$ & $151^{\mathrm{d}, \mathrm{e}}$ & $87^{e}$ & $598^{c}$ & $149^{\mathrm{d}, \mathrm{e}}$ & $106^{\mathrm{d}, \mathrm{e}}$ & $668^{c}$ & $204^{d}$ & $106^{\mathrm{d}, \mathrm{e}}$ & $1213^{\mathrm{a}}$ & $244^{\mathrm{d}}$ & $126^{\mathrm{d}, \mathrm{e}}$ & 74.4 & 0.000 & 0.000 & 0.000 \\
\hline NEI & $2136^{a, b}$ & $797^{c}$ & $544^{c}$ & $2172^{a, b}$ & $766^{c}$ & $524^{c}$ & $2393^{a}$ & $768^{c}$ & $523^{c}$ & $1915^{\mathrm{b}}$ & $647^{c}$ & $491^{c}$ & 152.4 & 0.000 & 0.000 & 0.000 \\
\hline RE:DEI & $58 \%^{\mathrm{b}}$ & $58 \%^{\mathrm{b}}$ & $50 \%^{\mathrm{c}}$ & $64 \%^{\mathrm{a}}$ & $54 \%^{\mathrm{b}, \mathrm{c}}$ & $45 \%$ d,e & $65 \%^{a}$ & $53 \%^{\mathrm{c}}$ & $47 \%{ }^{\mathrm{d}, \mathrm{e}}$ & $49 \%^{c, d}$ & $44 \%$ d,e & $43 \%^{e}$ & $1.5 \%$ & 0.000 & 0.000 & 0.014 \\
\hline
\end{tabular}

C, control; P, protein; L, lipid; S, starch; H, M and L, high, medium and low ration levels; D, R and D $\times$ R, $P$ values for effects of diet, ration or the interaction, respectively; FCR, feed conversion ratio; GPI, gross protein intake; FP, faecal protein: DPI dige energy; DEI, digestible energy intake; BUE, brachial and urinary energy; MEI, metabolisable energy intake; RE, retained energy; HP, heat production; HeE, basal metabolism; HiE, heat increment energy; NEI, net energy intake.

a,b,c,d,e,t Values within a row with unlike superscript letters were significantly different $(P<0.05)$. 


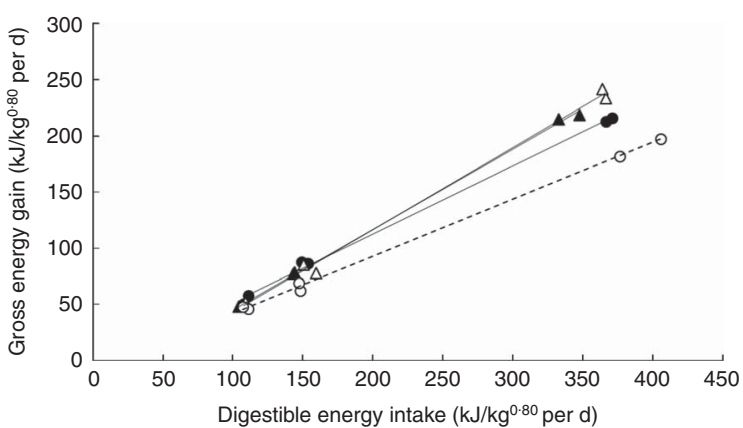

Fig. 1. Energy gain $\left(\mathrm{kJ}^{\mathrm{kg}} \mathrm{kg}^{0.8}\right.$ per $\left.\mathrm{d}\right)$ by barramundi when fed different rations of each experimental diet. The regression equation of each diet is also shown. There was no significant difference in the linear regressions between the control, protein and lipid diet treatments. The regression equation of fish fed the starch diet was significantly different from each of the other treatments. Control $\left(y=0.6073 x-8.6855, R^{2}=0.9974\right) ; \boldsymbol{\Delta}$, protein $(y=0.7148 x-26.324$, $\left.R^{2}=0.9988\right) ; \quad \triangle$, lipid $\left(y=0.7302 x-29.821, \quad R^{2}=0.9961\right) ; \quad O$, starch $\left(y=0.5078 x-8.8594, R^{2}=0.9985\right)$.

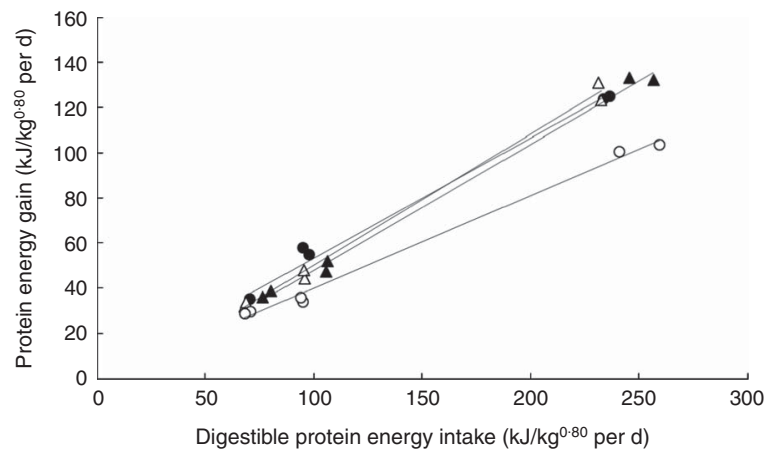

Fig. 2. Protein energy gain $\left(\mathrm{kJ} / \mathrm{kg}^{0.8}\right.$ per d) by barramundi when fed different rations of each experimental diet. The regression equation of each diet is also shown. There was no significant difference in the linear regressions between the control, protein and lipid diet treatments. The regression equation of fish fed the starch diet was significantly different from each of the other treatments. Control $\left(y=0.5335 x-0.0885, R^{2}=0.9865\right)$; $\mathbf{A}$, protein $(y=0.5565 x-7.6372$, $\left.R^{2}=0.996\right) ; \quad \triangle$, lipid $\left(y=0.5818 x-8.0943, R^{2}=0.9951\right) ; O$, starch $(y=$ $\left.0.4122 x-1.3025, R^{2}=0.9945\right)$.

$\left(\mathrm{g} / \mathrm{kg}^{0 \cdot 8}\right.$ per $\left.\mathrm{d}\right)$ for each diet were described by the following linear equations (Fig. 2):

$$
\begin{aligned}
& y_{S}=0.412( \pm 0.003) x-1.302\left( \pm 0.417 R^{2}=0.994\right. \\
& y_{L}=0.582( \pm 0.006) x-8.094( \pm 0.572), R^{2}=0.995 \\
& y_{P}=0.556( \pm 0.005) x-7.637( \pm 0.527), R^{2}=0.996 \\
& y_{C}=0.534( \pm 0.004) x-0.088( \pm 0.588), R^{2}=0.986
\end{aligned}
$$

The coefficient of utilisation was significantly lower for Diet S relative to each of the other diets. There was no difference in the protein energy utilisation coefficient $\left(k_{P E}\right)$ between Diets $\mathrm{P}$, $\mathrm{L}$ and $\mathrm{C}$.

There were also different levels of lipid energy retention between the starch diet and every other treatment (Table 3). This resulted in the coefficient of utilisation being significantly higher for Diet $\mathrm{S}$ relative to each of the other diets. However, Diet $\mathrm{P}$ also

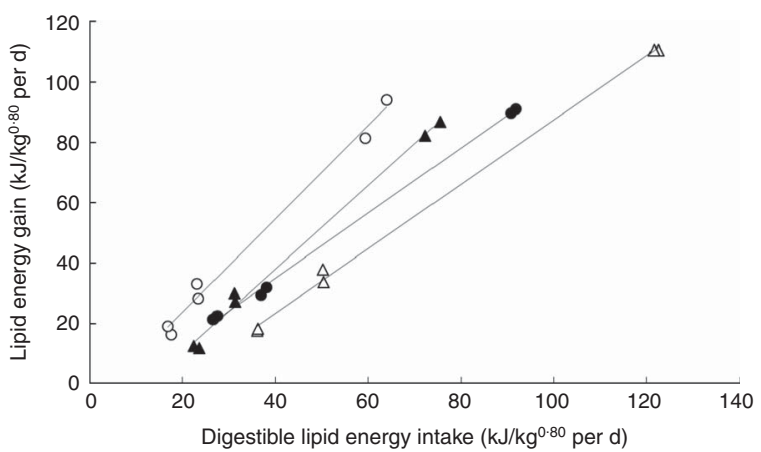

Fig. 3. Lipid energy gain $\left(\mathrm{kJ} / \mathrm{kg}^{0.8}\right.$ per $\left.\mathrm{d}\right)$ by barramundi when fed different rations of each experimental diet. The regression equation of each diet is also shown. There were no significant differences in the linear regressions among each of the control, protein, lipid and starch diet treatments. Control $\left(y=1.0812 x-8.375, R^{2}=0.9987\right) ; \Delta$, protein $(y=1.3873 x-17.558$, $\left.R^{2}=0.9942\right) ; \triangle$, lipid $\left(y=1.0699 x-19.619, R^{2}=0.9983\right) ; O$, starch $(y=1.5478 x-$ $\left.7.3317, R^{2}=0.9915\right)$.

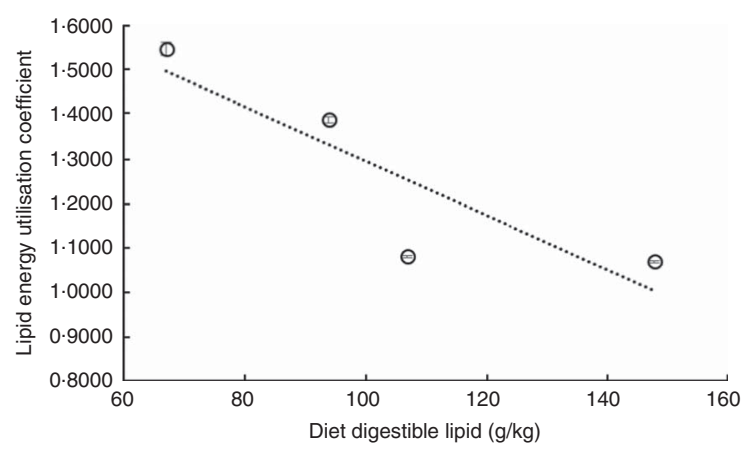

Fig. 4. Lipid energy utilisation coefficients relative to the dietary concentration of lipid $\left(y=-0.0061 x+1.9036, R^{2}=0.7618\right)$. Values are means with their standard errors.

had a significantly higher level of lipid energy utilisation relative to the lipid and control diets. The lipid energy utilisation efficiencies $\left(\mathrm{kJ} / \mathrm{kg}^{0.8}\right.$ per $\left.\mathrm{d}\right)$ for each diet were described by the following linear equations (Fig. 3):

$$
\begin{aligned}
& y_{S}=1.5478( \pm 0.015) x-7.332( \pm 0.500), R^{2}=0.991, \\
& y_{L}=1.070( \pm 0.002) x-19 \cdot 619( \pm 1.469), R^{2}=0.998, \\
& y_{P}=1.387( \pm 0.006) x-17.558( \pm 0.456), R^{2}=0.994, \\
& y_{C}=1.081( \pm 0.002) x-8.375( \pm 0.183), R^{2}=0.999 .
\end{aligned}
$$

When the lipid energy utilisation coefficients $\left(k_{L E}\right)$ were examined relative to the dietary concentration of lipid, a strong but non-significant $(P=0 \cdot 127)$ linear relationship was observed (Fig. 4).

\section{Determination of macronutrient component contributions to energy utilisation}

The different combinations of protein, lipid and starch among the diets in the present study allow for the analysis of the 
Table 4. Component energetic contributions from each macronutrient in each diet and the calculated and measured energetic parameters*

\begin{tabular}{|c|c|c|c|c|c|c|}
\hline \multirow[b]{2}{*}{ Diets } & \multirow[b]{2}{*}{ Parameters } & \multirow[b]{2}{*}{ Protein } & \multirow[b]{2}{*}{ Lipid } & \multirow[b]{2}{*}{ Starch } & \multicolumn{2}{|c|}{ Energy } \\
\hline & & & & & Calculated & Measured \\
\hline & Assumed energetic value $(\mathrm{MJ} / \mathrm{kg})$ & $23 \cdot 6$ & 38.5 & $17 \cdot 3$ & & \\
\hline \multirow[t]{4}{*}{ Control } & Digestible nutrient (g/kg) & 448 & 107 & 111 & & \\
\hline & Digestible energy $(\mathrm{M} J / \mathrm{kg})$ & 10.57 & $4 \cdot 12$ & 1.92 & $16 \cdot 61$ & $16 \cdot 61$ \\
\hline & Proportion of total energy (\%) & $63 \cdot 6$ & 24.8 & 11.6 & & \\
\hline & Utilisation coefficients & 0.534 & 0.821 & 0.438 & 0.594 & 0.607 \\
\hline \multirow[t]{4}{*}{ Protein } & Digestible nutrient (g/kg) & 545 & 94 & 19 & & \\
\hline & Digestible energy $(\mathrm{MJ} / \mathrm{kg})$ & $12 \cdot 86$ & 3.62 & 0.33 & $16 \cdot 81$ & $16 \cdot 70$ \\
\hline & Proportion of total energy (\%) & 76.5 & 21.5 & $2 \cdot 0$ & & \\
\hline & Utilisation coefficients & 0.557 & 1.345 & 0.438 & 0.715 & 0.715 \\
\hline \multirow[t]{4}{*}{ Lipid } & Digestible nutrient (g/kg) & 455 & 148 & 29 & & \\
\hline & Digestible energy $(\mathrm{M} / \mathrm{kg})$ & $10 \cdot 74$ & 5.70 & 0.50 & 16.94 & $16 \cdot 91$ \\
\hline & Proportion of total energy (\%) & 63.4 & 33.6 & 3.0 & & \\
\hline & Utilisation coefficients & 0.582 & 1.036 & 0.438 & 0.730 & 0.730 \\
\hline \multirow[t]{4}{*}{ Starch } & Digestible nutrient (g/kg) & 441 & 67 & 214 & & \\
\hline & Digestible energy (MJ/kg) & $10 \cdot 41$ & $2 \cdot 58$ & $3 \cdot 70$ & $16 \cdot 69$ & $16 \cdot 69$ \\
\hline & Proportion of total energy (\%) & $62 \cdot 4$ & $15 \cdot 5$ & $22 \cdot 2$ & & \\
\hline & Utilisation coefficients & 0.412 & 0.821 & 0.438 & 0.481 & 0.507 \\
\hline
\end{tabular}

component contributions of each macronutrient to energy retention (Table 4). This assumes that each macronutrient is contributing a part of the dietary energy proportional to its content in the diet, its energetic value and a component utilisation value.

On the basis of previously mentioned assumptions, each of the component energy utilisation values was derived using multiple regression analysis. For each of the diets, protein contribution can be defined by converting protein utilisation to protein energy utilisation, and thus defining the component protein energy utilisation (Fig. 2). Therefore, because we have a definitive assessment of protein energy utilisation efficiencies (see Equations (5)-(8)), we can also derive by multiple regression the remaining unknown variables, which constitute the contribution of both lipid energy and starch energy to the partial efficiency of energy utilisation in each diet (Tables 1 and 3). Although we have an assessment of the partial efficiency of lipid energy utilisation (Fig. 3), the fact that lipid energy gain in this representation also includes lipid deposited from non-lipid origins (i.e. starch and/or protein energy), it was necessary to derive the component lipid energy utilisation using multiple regression methods.

\section{Effect of macronutrient energy bias on protein, lipid and energy budgets}

There was a range of significant effects attributable to diet, feed ration level and the interaction term on the protein, lipid and energy budget parameters (Table 3). Exceptions to this were for the digestible protein intake (DPI), for which there were no significant interactions between diet and ration level. Gross protein intake (GPI) was highest in fish fed Diet $\mathrm{P}$ at ration level $\mathrm{H}$ with the corresponding lowest GPI at the same ration level for Diet L (Table 3). Faecal protein (FP) was also highest in fish fed Diet $\mathrm{P}$, and this was consistent across each of the ration levels. The lowest FP was observed in fish fed diet L, again across each of the ration levels. DPI was highest in fish fed Diet $\mathrm{P}$ at ration level $\mathrm{H}$, and although these differences were significant they were much smaller than those seen on GPI. Protein losses through BUN protein equivalent were highest among fish fed Diet $\mathrm{S}$ at ration level $\mathrm{H}$, although the differences at lower ration levels were less obvious. Retained protein (RP) at the highest ration levels was similar in C, P and L diet groups, but significantly poorer in Diet $\mathrm{S}$. The ratio of RP:DPI was highest among fish fed Diet $\mathrm{C}$ at ration level $\mathrm{M}$. At ration level $\mathrm{H}$, there was no significant difference among the RP:DPI for Diets C, P and L but for Diet S it was significantly lower (Table 3).

Gross lipid intake (GLI) was highest for fish fed Diet L at ration level $\mathrm{H}$ with the corresponding lowest GLI at the same ration level for Diet S (Table 3). Faecal lipid (FL) content was the highest among fish fed Diet $\mathrm{P}$, and this was consistent across the ration levels. The lowest FL, across the ration levels, was found in fish fed both Diets C and S. Digestible lipid intake (DLI) was the highest among fish fed Diet $\mathrm{L}$ at ration level $\mathrm{H}$, and for the other ration levels DLI was significantly higher compared with Diet L. Retained lipid (RL) at the highest ration levels was similar among fish fed diets C, P and S, but significantly higher among fish fed Diet L. The ratio of RL:DLI was the highest among fish fed Diet S, and this was consistent across ration levels. The ratio of RL:DLI was lowest among fish fed Diet $\mathrm{L}$, and this too was consistent across ration levels. The ratio between RL:RP for Diets L and S were similar and significantly higher compared with fish fed Diets $\mathrm{C}$ and $\mathrm{P}$. In most cases, this declined with declining ration, although no such effect was observed with Diet C (Table 3). 
Gross energy intake (GEI) was highest among fish fed Diet S at ration level $\mathrm{H}$ with the corresponding lowest GEI at the same ration level being from Diet $\mathrm{P}$ (Table 3). Among the lower ration levels, there were no significant differences in GEI. These differences were also reflected in the DEI across treatments. Faecal energy was highest among fish fed both Diet $\mathrm{C}$ and $\mathrm{S}$ and lowest among fish fed Diet P. BUE losses were highest among fish fed Diet $\mathrm{S}$ at ration level $\mathrm{H}$ and $\mathrm{M}$, although at the lowest ration level BUE was highest among fish fed Diet $P$. The highest MEI at ration level $\mathrm{H}$ was seen in fish fed Diet $\mathrm{S}$, but at the two lower ration levels it was higher from Diet C. Lowest MEI values were from Diet $\mathrm{P}$ and the highest ration level $(\mathrm{H})$, but at the two lower ration levels MEI intake was lower from Diet S. RE was highest among fish fed Diet $\mathrm{L}$ at ration level $\mathrm{H}$, and lowest among fish fed Diet $S$ at the lowest ration level, although the RE of fish fed Diet $S$ was the lowest within each of the ration levels. HP was highest, and substantially so, in fish fed Diet $S$ at ration level $\mathrm{H}$, although differences at lower ration levels were less obvious. HeE had significant effects attributable to both diet and ration, but not interaction. HiE was highest among fish fed Diet $\mathrm{S}$ at ration level $\mathrm{H}$, which was more than twice that of fish fed the same ration from Diet $\mathrm{P}$. This effect was reversed at the lower ration levels with higher HiE values observed from Diet $\mathrm{S}$ at the two lowest ration levels. Net energy intake (NEI) was highest among fish fed Diet L and lowest among fish fed Diet S. Ration also had a clear effect on NEI, although differences between fish fed Diets C, P and L at each of the ration levels were nominal. The NEI values of fish fed Diet $S$ were significantly lower at each ration level. The ratio of RE:DEI typically declined with declining ration. The RE:DEI values were similar between Diets P and L at similar ration levels, but significantly poorer when fed Diet $S$ at each ration level except the lowest one. Diet $\mathrm{C}$ was a little different to the other diets and showed a largely consistent RE:DEI across the ration levels and at a high level (>50\%) (Table 3 ).

\section{Discussion}

The present study sought to define the relative contributions of each of the three macronutrients (protein, lipid and starch) in supplying DE in diets fed to juvenile barramundi. This has enabled an insight into the roles that these macronutrients play in contributing to energy provision in this species. Understanding this relationship is critical to fish nutrition because of the strong intrinsic link between fish growth, energy demand and diet energy density.

\section{Effect of macronutrient energy bias on growth, feed utilisation and body composition}

By using diets with equivalent levels of DE but with differences in proportions of protein, lipid or starch providing that energy, clear effects were seen in this experiment. For each of these treatments, the strategy of feeding each diet at specific ration levels has allowed us to build substantially on earlier findings from using these same diets, which were previously fed over a longer term ${ }^{(12)}$. Therefore, in the present study, we focus our discussion on the effects within ration levels to allow us to examine the diet-specific effects. At the highest ration level, the responses of growth were generally consistent with the earlier study ${ }^{(12)}$. In the earlier study, the best growth was seen with Diet $\mathrm{P}$, whereas in the present study the best growth was seen with Diet L. However, in both studies, the poorest growth was seen with Diet S. At lower ration levels ( $M$ and $L$ ), growth was not consistent with the pattern seen at the $\mathrm{H}$ ration level. At lower ration levels, the best growth was seen among fish fed Diet P, followed by Diet L, and fish fed Diet S still performed the poorest. These results are directly comparable with those from our earlier study, and suggest that at the highest ration level, fed to apparent satiety, feed intake variability may have altered the responses. In another similar study by Saravanan et al. ${ }^{(11)}$ with rainbow trout fed either high- or low-protein diets with energy biased towards either starch or lipid, fish down-regulated their feed intake when fed starch-based diets. This observation was a direct contrast to the present study where barramundi increased their satietal intakes with starch-based diets. Differing again were the observations of Schrama et al. ${ }^{(14)}$, who observed in the omnivorous species tilapia that growth was not compromised with the use of starch as an energy source relative to that seen when lipid was used instead. We suggest that these differences are directly linked to the ability of tilapia to digest and utilise glucose from starch, whereas starch digestion by barramundi is comparatively poorer, and its ability to regulate blood glucose is questionable ${ }^{(25-27)}$. Clearly, there appears to be different nutritional capacity among different fish species to utilise starch as an energy source.

The responses of feed efficiency (FCR) to ration within each diet are consistent with observations of most studies on restricting nutrient/energy supply to fish, and the present findings are consistent in this regard with other findings from this species $^{(4,28)}$. An advantage of using this pair-feeding regimen is that it allows for a very clear examination of the effect of diet composition on performance criteria independent of feed intake variability. However, we do acknowledge that this does potentially cause complications in the application of digestibility values across variable feed intake levels. Some of the clearest implications from the variation in energy supply by different macronutrients can be seen by the cross-diet comparison of FCR at each of the two lower ration levels in the present study.

Effects of each of the diets on fish body composition were noted primarily in terms of the whole-body lipid, DM and protein concentrations. One of the most notable compositional effects at the highest ration level $(\mathrm{H})$ was the difference in lipid concentrations of fish fed Diet $\mathrm{L}$ relative to the other treatments, and that Diet P had the lowest lipid concentrations. These observations from the present study contrast those from an earlier study using these same diets, in that the lipid concentrations in fish fed Diet $\mathrm{S}$ are considerably lower and those of Diet $\mathrm{L}$ are higher ${ }^{(12)}$. At lower ration levels in the present study this effect of the diets with considerable starch content (Diet $\mathrm{C}$ and S) on lipid concentration in the body is more consistent with our earlier study. Reasons for this discrepancy at the satiety $(\mathrm{H})$ ration level are unclear. These present results (from the $\mathrm{H}$ ration) are, however, consistent with those of Schrama et al. ${ }^{(14)}$, who also noted higher levels of lipid in the 
whole body of fish (Tilapia) fed diets high in lipid, but less so in fish fed diets high in starch.

\section{Effects of macronutrient bias on energy utilisation}

The efficiency of energy utilisation (i.e. the ratio of gross energy gain as a function of DEI over a range of intake levels, expressed as $k_{E}$ ) differed among each of the treatments. In this study, the relationship between energy intake and gain was observed to be linear, with a calculated energy utilisation constant value that varied between $k_{E}=0.507$ and 0.730 , subject to diet. For Diet $\mathrm{C}$ (the most analogous to a commercial diet), $k_{E}=0.607$, which is generally consistent with other $k_{E}$ values that have been determined for this species ${ }^{(4,21)}$. In an earlier study ${ }^{(4)}$, a range in the values of $k_{E}$ of $0 \cdot 61-0.76$, with an average of 0.68 , has been determined and shown to be marginally affected by fish size. In a subsequent study, the $k_{E}$ values have also shown to be influenced by temperature, with $k_{E}$ values ranging from 0.42 to 0.59 and being lower outside optimal thermal regimens ${ }^{(29)}$

In the present study, a range of $k_{E}$ values was observed and clearly related to the variation in macronutrients used to supply equivalent levels of DE in each of the diets. Those diets higher in starch had poorer $k_{E}$ values, with Diet C (135 g/kg starch) $k_{E}=0.607$ and Diet S $\left(225 \mathrm{~g} / \mathrm{kg}\right.$ starch) $k_{E}=0.507$, compared with Diet P $(17 \mathrm{~g} / \mathrm{kg}$ starch $) k_{E}=0.715$ and Diet $\mathrm{L}(29 \mathrm{~g} / \mathrm{kg}$ starch) $k_{E}=0 \cdot 730$. A clear negative relationship between the $k_{E}$ values and diet starch concentration is seen (Fig. 5). Our findings in the present study are similar to those reported by Schrama et $a l .{ }^{(14)}$, who also reported a range in $k_{E}$ values when diets were biased to either starch $\left(k_{E}=0.561\right)$ or lipid $\left(k_{E}=\right.$ 0.663). A key difference between these studies was that in the present one we can isolate this effect from differences in DE concentration of the diets, and clearly ascribe the effects solely to macronutrient supply differences. Some significant differences in HEm were observed among the different diets. For those diets largely devoid of starch the HEm was estimated to be $36 \cdot 8-40 \cdot 8 \mathrm{~kJ} / \mathrm{kg}^{0.8}$ per $\mathrm{d}$, whereas those diets with starch had HEm values estimated at $14.3-17.4 \mathrm{~kJ} / \mathrm{kg}^{0.8}$ per $\mathrm{d}$. However, an important constraint is that these are estimated values derived from extension of the linear regression functions to their intercept of the $x$-axis, and given that there were no ration levels below the HEm values these estimations are beyond the bounds

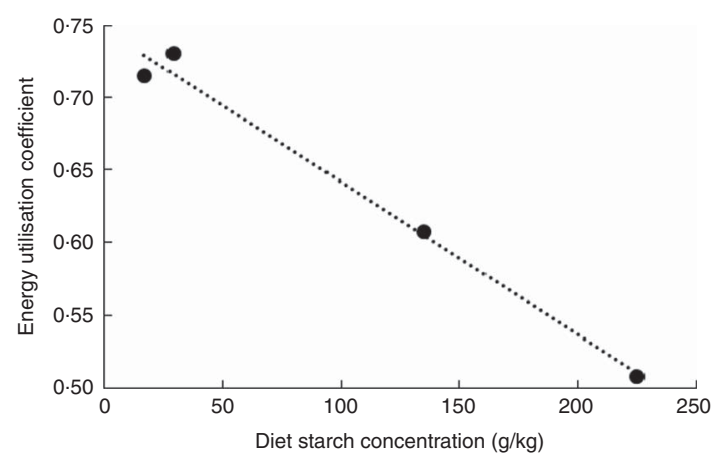

Fig. 5. Relationship between diet starch concentration and energy utilisation coefficient $\left(k_{E}\right)$ values $\left(y=-0.001 x+0.747, R^{2}=0.987\right)$. of the data. As such we suggest that these differences may be an artefact of the extrapolation of the data set.

\section{Effects of macronutrient bias on protein and lipid utilisation}

The protein utilisation efficiency was determined as the amount of dietary digestible protein $\left(\mathrm{g} / \mathrm{kg}^{0.7}\right.$ per $\left.\mathrm{d}\right)$ required to deposit a gram of protein in the body of the animal. In the present study values $\left(k_{P}\right)$ determined in the present study ranged from $k_{P}=0.412$ to 0.580 (data not shown). This compares well with values $\left(k_{P}=0.49-0.54\right)$ determined by Glencross ${ }^{(4)}$ and Glencross \& Bermudes ${ }^{(29)}$ for barramundi of different sizes and at different temperatures. The values also compare well with other carnivorous marine species like the European seabass (Dicentrarchus labrax) for which a value of $k_{P}=0.52$ was reported $^{(30)}$.

In the present study, a focus was made on the energy retention as protein energy retention. This was estimated based on its energy equivalent, in this case $23.6 \mathrm{~kJ} / \mathrm{g}$ protein, and expressed relative to the metabolic body weight $\left(\mathrm{W}^{0.8}\right)$ of the animal rather than its protein body weight $\left(\mathrm{W}^{0 \cdot 7}\right)^{(8)}$. The calculated energy cost as DE (kJ) for deposition of protein from each diet varied and was shown to be significantly higher with the inclusion of starch in the diet. The energy cost values $\left(1 / k_{P E}\right)$ determined in the present study for protein deposition ranged from 1.72 to $2.43 \mathrm{~kJ}$ per $\mathrm{kJ}$ of protein energy deposited, with higher cost values of $1.87-2.43$ being from diets higher in starch. This further supports that protein synthesis in the presence of higher dietary starch levels is more energetically expensive. This compares to other marine fish species (e.g. Sparus aurata, Dicentrarchus labrax and Epinephelus aeneus), and the freshwater carp (Cyprius carpio) which had $1 / k_{P E}$ values ranging from 1.78 to $1.90^{(8,31)}$.

Lipid utilisation efficiency (data not shown) was determined as the amount of digestible dietary lipid $\left(\mathrm{g} / \mathrm{kg}^{0.9}\right.$ per $\left.\mathrm{d}\right)$ required to deposit a gram of lipid in the body of the animal ${ }^{(21)}$. In the present study, lipid utilisation efficiency values $\left(k_{L}\right)$ ranged from $k_{L}=1.07$ to 1.55 . The utilisation of dietary lipid energy for lipid energy deposition to determine the partial efficiencies of $k_{L E}$ was also examined. What appeared unusual about these values is that they were all greater than 1 . This implied that there was greater lipid energy deposition than lipid energy intake, resulting in a NE gain from this macronutrient and clearly indicating synthetic activity. Although a similar scenario for protein would be impossible, for lipid it demonstrates that there is lipid being synthesised from other macronutrient substrates (e.g. starch or protein). From diets low in lipid, it can be noted that the relative contribution to lipid synthesis from these other macronutrients is enhanced.

The energy cost $\left(1 / k_{L E}\right)$ for lipid gain in the present study ranged from 0.65 to $0.93 \mathrm{~kJ}$ per $\mathrm{kJ}$ of lipid deposited. This was similar to the range of values $(0.83-0 \cdot 86)$ reported by Glencross et al. ${ }^{(32)}$ with rainbow trout (Oncorbynchus mykiss), but was substantially lower than that the $1 \cdot 10,1 \cdot 11$ and 1.31 reported by Lupatsch et al. ${ }^{(8)}$ for three marine species (Sparus aurata, Dicentrarchus labrax and Epinephelus aeneus). In carp, the efficiency was estimated at $1.39^{(31)}$, demonstrating that lipid accumulation from lipid energy intake was a highly efficient 
process in barramundi, similar to other carnivorous species ${ }^{(32)}$. That the energy cost of lipid gain is below one also demonstrates that this is an energetically efficient process in terms of energy storage. In contrast with the values of energy cost for protein deposition, which showed that the energetic cost for protein deposition was almost twice that of the energetic value for synthesis, lipid is so much more useful in terms of its storage mechanisms, because it uses less energy for storage than its own energetic value. One observation of note was the differences in the $1 / k_{L E}$ values, with Diet S having the lowest value of $1 / k_{L E}=0.65$, showing that lipid storage from starch is very efficient.

\section{Effects of macronutrient bias on component energy utilisation}

Energy retention in fish consists almost exclusively of protein or lipid deposition; therefore, the efficiency of energy gain in terms of protein and lipid gain can be considered separately using multiple regression analysis as described first by Kielanowski ${ }^{(33)}$. The comparison of the four diets in this study showed that the inclusion of starch in the diet had a significant effect on the gain of either protein or lipid relative to DEI, and a clear reduction in protein synthesis with the inclusion of this macronutrient in the diets.

When examining the components of energy utilisation, we have worked on the premise that it is the sum of the digestible value of protein, lipid and starch, their relative energetic proportions (\%) in the diet and a discrete component utilisation $\left(\theta k_{P E}, \theta k_{L E}\right.$ or $\left.\theta k_{S E}\right)$ of each macronutrient that combines to provide the overall $k_{E}$ value for any particular diet (Table 4). Using this premise, we observed that the component protein energy utilisation value $\left(\theta k_{P E}\right)$ was significantly impaired with the higher inclusion levels of dietary starch (Diet $S \theta k_{P E}=0.412$ cf. Diet L $\theta k_{P E}=0.582$ ). In diets with lower levels of digestible starch (e.g. Diet C $\theta k_{P E}=0.534 ; 111 \mathrm{~g} / \mathrm{kg}$ ), although a numerically lower $\theta k_{P E}$ was observed, it was not significantly reduced relative to diets with nominal levels of starch (e.g. Diet $\mathrm{P}$ $\left.\theta k_{P E}=0.557\right)$.

The component lipid energy utilisation value $\left(\theta k_{L E}\right)$ was highly variable compared with the other component energy utilisation values $\left(\theta k_{P E}\right.$ or $\left.\theta k_{S E}\right)$ for the other macronutrients, with $\theta k_{L E}$ values ranging from 0.821 to 1.345 (Table 4). These determined values appear to reflect both the inclusion of dietary starch (e.g. Diet S $\theta k_{L E}=0.821$ cf. Diet P $\theta k_{L E}=1.345$ ), and influences of dietary lipid level on the component lipid energy utilisation (e.g. Diet P $\theta k_{L E}=1.345$ cf. Diet L $\theta k_{L E}=1.036$ ). We suspect that the variability in this component utilisation value reflects the responsive nature of the metabolism of lipids by this species in response to variable nutrient supply. In effect, what we are observing is an enhanced capacity of the animal to produce lipid from protein energy sources. Although it is less efficient than that from lipid or protein, there is still substantial lipid synthesis from starch energy occurring.

The component starch energy utilisation values $\left(\theta k_{S E}\right)$ determined by using the multiple regression approach were determined to be the same across all diets $\left(\theta k_{S E}=0.438\right)$.
Energy deposition from starch was clearly the least efficient of all the macronutrients (although a poorer $\theta k_{P E}$ was noted for Diet S). We suggest that barramundi has limited metabolic capacity to utilise starch-derived energy. Although it can produce lipids from glucose precursors, it clearly does so at a less-efficient rate than that seen from either protein or lipid directly.

\section{Conclusions}

The results of this study show that barramundi have clear metabolic inefficiencies associated with the inclusion of starch in their diet. With the increasing inclusion of starch in the diet of this species there was a reduction in the efficiency of protein (protein energy) utilisation and this contributed to an overall decline in the efficiency of energy utilisation. In the absence of starch, protein utilisation was constant and it was unaffected by its concentration in the diet. Collectively, the findings of this study support the notion that the concentration and type of macronutrient mix in a diet for barramundi has a significant effect on the ability of the fish to use those nutrients for energy. This finding suggests the existence of a metabolic mechanism that influences the ability of fish to utilise discrete nutrients for energy, independent of total energy intake.

\section{Acknowledgements}

The authors acknowledge the technical support of Nick Polymeris and Mike Anderson.

This study was supported by the Australian Centre for International Agricultural Research, Project FIS-2006-141.

B. D. G., D. B., S. I. and N. M. W. all contributed to the experimental design. B. D. G., D. B., S. C., N. B. and S. I. all contributed to the conduct of the experiment. D. B. manufactured the diets, N. B. and S. C. undertook most of the sample analysis. B. D. G. undertook the analysis of the data. B. D. G., S. I. and N. M. W. all contributed to the interpretation of the data and writing of the manuscript.

None of the authors has any conflicts of interest to declare.

\section{References}

1. Glencross BD (2006) Nutritional management of barramundi, Lates calcarifer - a review. Aquacult Nutr 12, 291-309.

2. Williams KC, Barlow CG, Rodgers L, et al. (2003) Asian seabass Lates calcarifer perform well when fed pellet diets high in protein and lipid. Aquaculture 225, 191-206.

3. Williams KC, Barlow C, Rodgers L, et al. (2006) Dietary composition manipulation to enhance the performance of juvenile barramundi (Lates calcarifer Bloch) reared in cool water. Aquacult Res 37, 914-927.

4. Glencross BD (2008) A factorial growth and feed utilisation model for barramundi, Lates calcarifer based on Australian production conditions. Aquacult Nutr 14, 360-373.

5. Glencross BD \& Bermudes M (2012) Using a bioenergetic modelling approach to understand the implications of heat stress on barramundi (Lates calcarifer) growth, feed utilisation and optimal protein and energy requirements - options for adapting to climate change? Aquacult Nutr 18, 411-422. 
6. Boujard T \& Medale F (1994) Regulation of voluntary feed intake in juvenile rainbow trout fed by hand or by self-feeders with diets containing two different protein/energy ratios. Aquat Liv Res 7, 211-215.

7. Catacutan MR \& Coloso RM (1995) Effect of dietary protein to energy ratios on growth, survival, and body composition of juvenile Asian seabass, Lates calcarifer. Aquaculture 131, 125-133.

8. Lupatsch I, Kissil GW \& Sklan D (2003) Comparison of energy and protein efficiency among three fish species Sparus aurata, Dicentrarchus labrax and Epinephelus aeneus: energy expenditure for protein and lipid deposition. Aquaculture 225, 175-189.

9. Hua K, Birkett S, De Lange CFM, et al. (2010) Adaptation of a non-ruminant nutrient-based growth model to rainbow trout (Oncorbynchus mykiss Walbaum). J Agric Sci 148, 17-29.

10. Dumas A, de Lange CFM, France J, et al. (2007) Quantitative description of body composition and rates of nutrient deposition in rainbow trout (Oncorbynchus mykiss). Aquaculture 273, 165-181.

11. Saravanan S, Schrama JW, Figueirdo-Silva A, et al. (2012) Constraints on energy intake in fish: the link between diet composition, energy metabolism, and energy intake in rainbow trout. PLOS ONE 7, e34743.

12. Glencross BD, Blyth D, Bourne N, et al. (2014) An analysis of the effects of different dietary macronutrient energy sources on the growth and energy partitioning by juvenile barramundi, Lates calcarifer, reveal a preference for proteinderived energy. Aquacult Nutr 20, 583-594.

13. Azevedo PA, van Milgen J, Leeson S, et al. (2005) Comparing efficiency of metabolisable energy utilisation by rainbow trout (Oncorbynchus mykiss) and Atlantic salmon (Salmo salar) using factorial and multivariate approaches. J Anim Sci 83, $1-11$.

14. Schrama JW, Saravanan S, Geurden I, et al. (2012) Dietary nutrient composition affects digestible energy utilisation for growth: a study on Nile tilapia (Oreochromis niloticus) and a literature comparison across fish species. BrJ Nutr 108, 277-289.

15. Sa R, Pousao-Ferreira P \& Oliva-Teles A (2007) Growth performance and metabolic utilization of diets with different protein:carbohydrate ratios by white sea bream (Diplodus sargus, L.) juveniles. Aquacult Res 38, 100-105.

16. Enes P, Panserat S, Kaushik S, et al. (2009) Nutritional regulation of hepatic glucose metabolism in fish. Fish Physiol Biochem 35, 519-539.

17. Lansard M, Panserat S, Plagnes-Juan E, et al. (2010) Integration of insulin and amino acid signals that regulate hepatic metabolism-related gene expression in rainbow trout: role of TOR. Amino Acids 39, 801-810.

18. Helland S, Grisdale-Helland B \& Nerland S (1996) A simple method for the measurement of daily feed intake of groups of fish in tanks. Aquaculture 139, 156-163.
19. Association of Official Analytical Chemists (2005) Official Methods of Analysis of the Association of Official Analytical Chemists, 15th ed. Washington, DC: AOAC.

20. Blyth D, Tabrett SJ \& Glencross BD (2014) Comparison of faecal collection methods and diet acclimation times for the measurement of digestibility coefficients in barramundi (Lates calcarifer). Aquacult Nutr 21, 248-255.

21. Glencross BD \& Bermudes M (2011) Effect of high water temperatures on energetic allometric scaling in barramundi (Lates calcarifer). Comp Biochem Physiol A Mol Integr Physiol 159, 167-174.

22. Salini MJ, Poppi DA, Turchini GM \& Glencross BD (2016) Defining the allometric relationship between size and nutrient turnover in barramundi Lates calcarifer. Comp Biochem Physiol A Mol Integr Physiol 201, 79-86.

23. Bureau DP, Kaushik SJ \& Cho CY (2002) Bioenergetics. In: Fish Nutrition, 3rd ed., pp 2-61 [JE Halver and RW Hardy, editors]. San Diego, CA: Academic Press.

24. Steel GD \& Torrie JH (1980) Principles and Procedures of Statistics - A Biometrical Approach, 2nd ed. Singapore: McGraw Hill Publishing.

25. Stone DAJ (2003) Dietary carbohydrate utilisation by fish. Rev Fish Sci 11, 337-369.

26. Glencross BD, Blyth D, Tabrett SJ, et al. (2012) An examination of digestibility and technical qualities of a range of cereal grains when fed to juvenile barramundi (Lates calcarifer) in extruded diets. Aquacult Nutr 18, 388-399.

27. Wade NM, Skiba-Cassy S, Dias K, et al. (2013) Postprandial molecular responses in the liver of the barramundi, Lates calcarifer. Fish Physiol Biochem 40, 427-443.

28. Bermudes M, Glencross BD, Austen K, et al. (2010) Effect of high water temperatures on nutrient and energy retention in barramundi (Lates calcarifer). Aquaculture 306, 160-166.

29. Glencross BD \& Bermudes M (2010) Effect of high water temperatures on the utilisation efficiencies of energy and protein by juvenile barramundi, Lates calcarifer. Fish Aquacult J 14, 1-11.

30. Lupatsch I, Kissil GW \& Sklan D (2001) Optimization of feeding regimes for European sea bass Dicentrarchus labrax: a factorial approach. Aquaculture 202, 289-302.

31. Schwarz FJ \& Kirchgessner M (1995) Effects of different diets and levels of feeding on retention and efficiency of utilization of energy and protein by carp (Cyprinus carpio). J Appl Ichthyol 11, 363-366.

32. Glencross BD, Hawkins WE, Evans D, et al. (2008) Evaluation of the influence of Lupinus angustifolius kernel meal on dietary nutrient and energy utilisation efficiency by rainbow trout (Oncorbynchus mykiss). Aquacult Nutr 14, 129-138.

33. Kielanowski J (1965) Estimates of the energy cost of protein deposition in growing animals. In Proceedings of the $3 \mathrm{rd}$ Symposium on Energy Metabolism, pp. 13-20 [Blaxter, K.L., editor]. London: Academic Press. 